\title{
О понятии конечного множества в конструктивной математике ${ }^{1}$
}

\author{
А. А. ВлАдИмиРов
}

\begin{abstract}
It is known that the concept of non-infinite set is nonmonolithic from the point of view of A.A. Markov's constructive mathematics. In the paper we present one general approach to constructivist conception of «type of non-infiniteness».
\end{abstract}

Ключевые слова: конструктивная математика, марковский конструктивизм, конечное множество

\section{1 Введение}

1. С интуитивной точки зрения, факт конечности того или иного множества объектов сводится к возможности перечислить эти объекты посредством некоторого списка. Например, конечность множества натуральных чисел, заданного условием

$$
\ll n=0 \text { или } n=1 »,
$$

устанавливается предъявлением списка $\{0,1\}$, исчерпывающего все - с точностью до равенства - элементы указанного множества. Тем самым свойство конечности множества относится к числу свойств общего вида

«существует вспомогательный объект $A$, нахо-

(1) дящийся в некотором заранее известном отношении к рассматриваемому объекту $X »$.

С точки зрения «классических» логики и математики, суждения вида (1) ничем не выделяются среди прочих. Напротив, с точки зрения марковского конструктивного направления при

\footnotetext{
${ }^{1}$ Работа поддержана РФФИ, проект № 09-06-00125.
} 
попытке понимания таких суждений встает принципиальный вопрос о характере упоминаемого в них «существования». Ситуация, когда известен метод явного построения искомого вспомогательного объекта, - как раз и отвечающая интуитивным представлениям, - не считается в его рамках равнозначной ситуации, когда имеется лишь логическое опровержение предположения о невозможности такого объекта. Соответственно математические понятия, определения которых имеют вид (1), в рамках марковского конструктивизма подвержены эффекту «расщепления». В качестве хорошо известного [6, гл. 2] примера здесь можно указать на «классическое» определение вещественного числа по Г. Кантору, перенесение которого в конструктивный математический анализ приводит к несовпадающим понятиям «конструктивного вещественного числа» и «псевдочисла». Представление о конечности множества не является в этом смысле исключением.

Согласно сообщениям [10, 9], уже в 1950-е годы А.А. Марковым было указано на наличие, самое меньшее, четырех несовпадающих конструктивных истолкований представления о конечности, которые были связаны им с понятиями финитного, субфинитного, квазифинитного и неинфинитного множества. Аналогичная проблематика (хотя и несколько иначе трактуемая) возникла также в «классической» теории рекурсии. Например, в известной монографии $[11, \S 5.6]$ используются способы задания конечного множества посредством канонических индексов ${ }^{2}$, а также посредством гёделевых номеров, разрешающих или перечисляющих эти множества рекурсивных функций ${ }^{3}$. Указанному вопросу уделялось также внимание и в рамках математического интуиционизма [1]. Целью настоящей статьи является развитие одного общего подхода к понятию «тип конечности множества» с точки зрения марковского конструктивного направления в математике. Некоторые из излагаемых далее результатов были анонсированы в заметке [2].

2. В качестве точных языков, применяемых для записи и истолкования математических суждений, мы намерены использовать языки ступенчатой семантической системы А.А. Маркова. В

\footnotetext{
${ }^{2}$ Это отвечает «финитным» множествам А.А. Маркова.

${ }^{3}$ Очерченная в [9] схема таких типов конечности не содержит.
} 
частности, мы сохраняем применявшуюся самим автором этой системы [7] польскую запись логических связок. Более подробное описание используемых обозначений может быть найдено в монографии [4].

Термин «множество» будет далее рассматриваться в качестве сокращения для словосочетания «формула языка $Я_{\omega+1}[4, \S 27]$, не имеющая отличных от $\mid \nabla$ параметров». Множества, задаваемые посредством формул языка $Я_{1}[4, \S 8]$, мы будем называть порождаемыми.

Өлементом произвольно фиксированного множества $\mathfrak{A}$ будет считаться всякий постоянный терм $[4, \S 6]$, подстановка которого в формулу $\mathfrak{A}$ вместо переменной $\mid \diamond$ приводит к верной замкнутой формуле. Ввиду возможности кодирования конструктивных объектов любой известной природы постоянными термами, указанные соглашения не влекут существенного ограничения общности рассмотрения.

\section{2 Основные определения}

1. Под списком постоянных термов естественным образом понимается постоянный терм, рассматриваемый как перечень своих

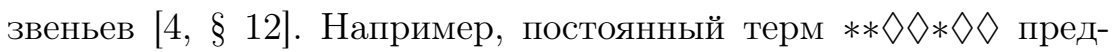
ставляет собой с указанной точки зрения список с членами $* \diamond \diamond$ и $\diamond$. Порождаемое множество [4, § $12.4(1)]$ пар *ut вида «постоянный терм $t$ есть член списка $u \gg$ мы в дальнейшем будем обозначать символом $\mathfrak{L}$.

2. Введенные А.А. Марковым понятия финитного, субфинитного, квазифинитного и неинфинитного множества могут быть определены следующим образом:

2.1. Множество $\mathfrak{A}$ называется финитным, если верна замкнутая формула

$$
\exists \sharp_{2} \forall\left|\diamond \equiv \mathfrak{A} \exists \sharp_{3} \&=\sharp_{3} * \sharp_{2}\right| \diamond\left[\mathfrak{L} \sharp_{3} \varepsilon .\right.
$$

Иначе говоря, финитность множества $\mathfrak{A}$ означает, что известен способ построения списка, членами которого являются все элементы множества $\mathfrak{A}$, и только они. 
2.2. Множество $\mathfrak{A}$ называется суббинитным, если верна замкнутая формула

$$
\exists \sharp_{\mathbf{2}} \forall\left|\diamond \supset \mathfrak{A} \exists \sharp_{\mathbf{3}} \&=\sharp_{\mathbf{3}} * \sharp_{\mathbf{2}}\right| \diamond\left[\mathfrak{L} \sharp_{\mathbf{3}}{ }^{\varepsilon} .\right.
$$

Иначе говоря, субфинитность множества $\mathfrak{A}$ означает, что известен способ построения списка, членами которого являются все элементы множества $\mathfrak{A}-$ но, возможно, не только они.

2.3. Множество $\mathfrak{A}$ называется квазифинитным, если верна замкнутая формула

$$
\exists \sharp_{3} \neg \forall \sharp_{2} \supset \mathfrak{C} \neg \forall\left|\diamond \equiv \mathfrak{A} \exists_{3} \&=\sharp_{3} * \sharp_{2}\right| \diamond\left[\mathfrak{L} \sharp_{3}{ }^{\varepsilon},\right.
$$

где $\mathfrak{C}-$ формула языка $Я_{1}$ вида

$$
\begin{aligned}
& \text { (1) } \exists\left|\diamond \& \exists \sharp_{4}=\right| \diamond * * \sharp_{2} \sharp_{3} \sharp_{4} \forall|\diamond<| \diamond \vee \vee=\left|\diamond \diamond \exists \sharp_{3}=\right| \diamond * * \diamond \sharp_{3} \diamond \\
& \exists \sharp_{2} \exists \sharp_{3} \exists \sharp_{4} \exists \sharp_{5} \exists \sharp_{6}=\mid \triangleright * * * \sharp_{5} \sharp_{2} * \sharp_{6} \sharp_{3} * * \sharp_{2} \sharp_{3} \sharp_{4},
\end{aligned}
$$

выражсаюая отношение «число звенъев списка, являющегося значением переменной $\sharp_{\mathbf{2}}$, не превосходит таковое для списка, являющегося значением переменной $\sharp_{3} »$.

Иначе говоря, квазифинитность множества $\mathfrak{A}$ означает известность способа построения натурального числа $n$, для которого будет опровергаться приведением к нелепости предположение о невозможности списка элементов множества $\mathfrak{A}$ среди списков с не более чем $n$ членами.

2.4. Множество $\mathfrak{A}$ называется неинфинитным, если верна замкнутая формула

$$
\neg \forall \sharp_{2} \neg \forall\left|\diamond \equiv \mathfrak{A} \exists \sharp_{3} \&=\sharp_{3} * \sharp_{2}\right| \diamond\left[\mathfrak{L} \sharp_{3} \varepsilon .\right.
$$

Иначе говоря, неинфинитность множества $\mathfrak{A}$ означает возможность привести к нелепости предположение о невозможности списка, членами которого являлись бы все элементы множества $\mathfrak{A}$, и только они. 
3. Как несложно заметить, каждое из определений $2.2,2.3$ и 2.4 имеет структуру вида

«осуществим вспомогательный объект $A$, для которого список $L$ элементов рассматриваемо-

(1) го множества квазиосуществим среди списков, находящихся к объекту $A$ в некотором заранее известном отношении».

Исключением является определение 2.1 - в случае существенно проблемного $[5, \S 1]$, т. е. удовлетворяющего условию

$$
\neg \forall \mid \diamond \supset \neg \neg \mathfrak{A} \mathfrak{A},
$$

множества $\mathfrak{A}$. Попытка переформулирования указанного определения в виде (1) давала бы утверждение о финитности множества $\neg \neg \mathfrak{A}$, а не множества $\mathfrak{A}$. Однако для нормальных 4 множеств определение финитности также подпадает под указанную схему, что позволяет рассматривать последнюю в качестве основы для общего определения типа конечности.

4. Понятие типа конечности множеств мы вводим следующим образом:

4.1. Множество т называется типом конечности, если для любого постоянного терма $t$ осуществим постоянный терм и, удовлетворяющий соотношению $*$ $и \in \mathfrak{T}$.

Для произвольно фиксированного типа конечности $\mathfrak{T}$ мы вводим следующее понятие T-конечного множества:

4.2. Пусть $\mathfrak{T}-$ тип конечности множеств. Множество $\mathfrak{A}$ называется Т-конечным, если осуществим постоянный терм и со свойством

$$
\neg \forall \sharp_{\mathbf{2}} \forall|\diamond \supset \&=| \diamond * u \sharp_{\mathbf{2}} \mathfrak{T} \neg \forall\left|\diamond \equiv \mathfrak{A} \exists_{\mathbf{3}} \&=\sharp_{\mathbf{3}} * \sharp_{\mathbf{2}}\right| \diamond\left[\mathfrak{L} \sharp_{\mathbf{3}}{ }^{\varepsilon} .\right.
$$

Иначе говоря, $\mathfrak{T}$-конечность множества $\mathfrak{A}$ означает осуществимость указания постоянного терма $u$, для которого список элементов $\mathfrak{A}$ квазиосуществим среди постоянных термов $t$ со свойством $* u t \in \mathfrak{T}$.

\footnotetext{
${ }^{4}$ То есть заданных средствами языка $Я_{\omega}$ и потому равнообъемных своим вторым отрицаниям.
} 
Типы конечности множеств, описанные в пункте 2 , допускают в рамках изложенной схемы следующую характеризацию:

4.3. Пусть Т - тип конечности вида

$$
\exists \sharp_{2}=\mid \diamond * \sharp_{2} \sharp_{2} \text {. }
$$

Тогда T-конечными являются множества с финитными вторыми отрицаниями, и только они.

4.4. Пусть т - тип конечности вида

$$
\exists \sharp_{2} \exists \sharp_{3} \&=\mid \triangleright * \sharp_{2} \sharp_{3} \forall \sharp_{3}<\sharp_{3} \vee=\sharp_{3} \diamond \sharp_{4} \exists \sharp_{5} \&=\sharp_{3} * \sharp_{4} \sharp_{5}\left[\mathfrak{L} * \sharp_{2} \sharp_{4} \varepsilon .\right.
$$

Тогда Т-конечными являются суббинитные множества, $и$ только они.

4.5. Пусть $\mathfrak{T}$ - тип конечности вида

$$
\exists \sharp_{2} \exists \sharp_{3} \&=\mid \diamond * \sharp_{3} \sharp_{2} \mathfrak{C},
$$

где через $\mathfrak{C}$ обозначена формула 2(1). Тогда T-конечными являются квазифинитные множества, и только они.

4.6. Пусть $\mathfrak{T}-$ тип конечности вида $=\triangleright \triangleright$. Тогда $\mathfrak{T}$-конечными являются неинфинитные множества, и только они.

\section{3 Сравнение типов конечности}

1. Тип конечности $\mathfrak{T}$ естественно считать мажорирующим тип конечности $\mathfrak{S}$, если любое $\mathfrak{T}$-конечное множество является также и $\mathfrak{S}$-конечным. Используемая нами семантика языка $Я_{\omega+1}$ не позволяет, однако, считать осмысленными условия, содержащие неограниченный теоретико-множественный квантор общности. Поэтому вышеописанное представление о мажорировании типов конечности нуждается в уточнении. Стандартным способом здесь является наложение ограничений на высоту рассматриваемых множеств:

1.1. Тип конечности $\mathfrak{T}$ называется $n$-мажсорирующим тип конечности $\mathfrak{S}$, если любое нормальное $\mathfrak{T}$-конечное множество не превосходящей $n$ высоты является также $\mathfrak{S}$-конечным.

Далее факт $n$-мажорирования типа конечности $\mathfrak{S}$ типом конечности $\mathfrak{T}$ мы будем обозначать символом $\mathfrak{T} \succcurlyeq_{n} \mathfrak{S}$. 
2. В дальнейшем два постоянных терма мы будем называть равносоставленными, если каждое звено одного из них является также звеном второго. Кроме того, мы введем следующее представление о разрешимости типа конечности:

2.1. Тип конечности T⿱ называется разрешимым, если множество т является порождаемым, а также может быть указано порождаемое множество Т', удовлетворяющее следующим условиям:

2.1.1. Для любых постоянного терма и и двух равносоставленных постоянных термов $t$ u $s$ невозможно одновременное выполнение соотношений $* u t \in \mathfrak{T} u * u s \in \mathfrak{T}^{\prime}$.

2.1.2. Для любых двух постоянных термов и и $t$ найдется равносоставленный $c t$ постоянный терм $s$, удовлетворяющий соотношению $*$ иs $\in \mathrm{V} \mathfrak{T T}$.

В частности, несложным образом устанавливается разрешимость всех типов конечности § 2.4.3-§ 2.4.6.

3. Основной целью настоящего параграфа является доказательство следующего утверждения:

3.1. Пусть $\mathfrak{T}$ и $\mathfrak{S}-$ - ва разрешимых типа конечности множеств. Тогда при любом $n \geqslant 1$ для выполнения соотношения $\mathfrak{T} \succcurlyeq_{n} \mathfrak{S}$ необходимо и достаточно, чтобы для любого постоянного терма и, удовлетворяющего условию

$$
\exists \sharp_{2} \exists|\triangleright \&=| \diamond * u \sharp_{2} \mathfrak{T},
$$

был осуществим такой постоянный терм $v$, что для любого постоянного терма $t$ со свойством $*$ и $\in \mathfrak{T}$ найдется равносоставленный ему постоянный терм s со свойством $* v s \in \mathfrak{S}$.

Доказательство. Достаточность сформулированного условия очевидна. Поэтому для завершения доказательства утверждения 3.1 остается установить необходимость этого условия.

В дальнейших рассуждениях постоянный терм $u$ со свойством (1) мы будем предполагать зафиксированным.

Обозначим через $(t: x \Rightarrow y)$ формулу языка $Я_{1}$, выражающую отношение «постоянный терм $t$ есть перевод $[4, \S 13]$ схемы $[8$, $\S 27]$ нормального алгорифма над алфавитом $\diamond *$, перерабатывающего значение переменной $x$ в значение переменной $y$ ». Далее, 
обозначим через $\mathfrak{S}^{\prime}$ порождаемое множество, отвечающее разрешимому типу конечности $\mathfrak{S}$ согласно определению 2.1. Наконец, обозначим через $\mathcal{A}$ нормальный алгорифм над алфавитом $\diamond *$, применимый к произвольно фиксированному постоянному терму $v$ в том и только том случае, когда осуществимы два равносоставленных постоянных терма $t$ и $s$ со свойствами $* u t \in \mathfrak{T}$ и $* v s \in \mathfrak{S}^{\prime}$. При этом будет предполагаться, что значением $\mathcal{A}(v)$ всегда является постоянный терм, удовлетворяющий соотношению $* u \mathcal{A}(v) \in \mathfrak{T}$ и обладающий равносоставленным постоянным термом $s$ со свойством $* v s \in \mathfrak{S}^{\prime}$.

Зафиксируем произвольным образом некоторый постоянный терм $r$ со свойством $* u r \in \mathfrak{T}$, и сопоставим каждому постоянному терму $w$ имеющее высоту 1 множество $\mathcal{K}(w)$ вида

$$
\begin{aligned}
& \mathcal{K}(w) \rightleftharpoons \& \vee \exists \sharp_{\mathbf{2}} \&=\sharp_{\mathbf{2}} * r \mid \nabla\left[\mathfrak{L}_{\mathbf{2}}{ }^{\varepsilon} \exists \sharp_{\mathbf{2}} \exists \sharp_{\mathbf{3}} \mathfrak{K}_{w}\right. \\
& \supset \exists_{\mathbf{2}} \exists \sharp_{3} \mathfrak{K}_{w} \exists \sharp_{2} \exists \sharp_{3} \& \mathfrak{K}_{w} \exists \sharp_{2} \&=\sharp_{2} * \sharp_{3} \mid \diamond\left[\mathfrak{L} \sharp_{2} \varepsilon,\right.
\end{aligned}
$$

где положено

$$
\mathfrak{K}_{w} \rightleftharpoons \exists|\diamond \& \&=| \nabla w\left(w: \mid \diamond \Rightarrow \sharp_{2}\right)\left(\left[\mathcal{A}^{\tau}: \sharp_{2} \Rightarrow \sharp_{3}\right) .\right.
$$

В том случае, когда постоянный терм $w$ является переводом схемы самоприменимого 5 нормального алгорифма, результат самоприменения которого представляет собой постоянный терм $v$ из области применимости алгорифма $\mathcal{A}$, элементы множества $\mathcal{K}(w)$ перечисляются списком $\mathcal{A}(v)$. В противном случае они перечисляются списком $r$. Соответственно, при любом выборе постоянного терма $w$ среди постоянных термов $t$ со свойством $* u t \in \mathfrak{T}$ квазиосуществим список элементов множества $\mathcal{K}(w)$.

Предположим теперь, что выполняется соотношение $\mathfrak{T} \succcurlyeq_{n} \mathfrak{S}$. Это означает осуществимость нормального алгорифма $\mathcal{B}$ над алфавитом $\diamond *$, применимого к переводу любого множества $\mathfrak{A}$ не превосходящей $n$ высоты, список элементов которого квазиосуществим среди постоянных термов $t$ со свойством $* u t \in \mathfrak{T}$, и перерабатывающего указанный перевод в такой постоянный терм $v$, что список элементов множества $\mathfrak{A}$ квазиосуществим среди постоянных термов $s$ со свойством $* v s \in \mathfrak{S}$. Тогда, согласно вышесказанному, осуществим нормальный алгорифм $\mathcal{C}$ над алфавитом $\diamond *$, перерабатывающий любой постоянный терм $w$ в такой

\footnotetext{
${ }^{5}$ То есть применимого к переводу своей схемы.
} 
постоянный терм $v$, что среди постоянных термов $s$ со свойством $* v s \in \mathfrak{S}$ квазиосуществим список элементов множества $\mathcal{K}(w)$.

По своему построению алгорифм $\mathcal{C}$ является самоприменимым, причем результатом его самоприменения является такой постоянный терм $v$, что среди постоянных термов $s$ со свойством $* v s \in \mathfrak{S}$ квазиосуществим список элементов множества $\mathcal{K}\left(\left[\mathcal{C}^{\tau}\right)\right.$. Однако последнее, по построению алгорифмов $\mathcal{K}$ и $\mathcal{A}$, автоматически означает неприменимость алгорифма $\mathcal{A}$ к постоянному терму $v$. Тем самым для любого постоянного терма $t$ со свойством $* u t \in \mathfrak{T}$ найдется равносоставленный ему постоянный терм $s$ со свойством $* v s \in \mathfrak{S}$, что и означает справедливость доказываемого утверждения.

Q.E.D.

4. В качестве простейшего приложения утверждения 3.1 приведем основанные на нем доказательства результатов А.А. Маркова о сравнении типов конечности $\S 2.2 .1-\S 2.2 .4$.

Рассмотрим постоянный терм $u \rightleftharpoons * * \diamond \diamond * \diamond \diamond$. Легко видеть, что для любого постоянного терма $v$ найдется постоянный терм $t$, не равносоставленный с $v$, но не обладающий звеньями, не являющимися звеньями списка $u$. Тем самым, утверждение о финитности любого субфинитного множества является неверным.

Рассмотрим постоянный терм $u \rightleftharpoons * \diamond \diamond$. Легко видеть, что для любого постоянного терма $v$ найдется постоянный терм $t$, обладающий ровно одним не входящим в $v$ звеном. Тем самым, утверждение о субфинитности любого квазифинитного множества является неверным.

Рассмотрим постоянный терм $u \rightleftharpoons \diamond$. Легко видеть, что для любого постоянного терма $v$ найдется постоянный терм $t$, число попарно графически различных звеньев которого превышает число звеньев $v$. Тем самым утверждение о квазифинитности любого неинфинитного множества является неверным.

Отметим, что неверность утверждения о финитности всех неинфинитных множеств может также быть выведена из теоремы $[11$, гл. 5, Теорема XV (b)].

\section{4 Дополнительные замечания}

1. Одним из математических понятий, определение которого опирается на понятие конечного множества, является понятие 
иммунного множества. С точки зрения вышеизложенной схемы, указанное определение может быть сформулировано - при конструктивном понимании математических суждений - следующим образом:

1.1. Инфинитное множество $\mathfrak{A}$ называется иммунным, если неверно, что для любого натурального числа $n$ найдется финитное множество $\mathfrak{A}_{n} \subseteq \mathfrak{A}$, содержащее не менее $n$ попарно различных элементов.

Одна из форм $[11$, гл. 9, Теорема XV] определения понятия гипериммунного множества при этом легко переписывается следующим образом:

1.2. Инфинитное множество $\mathfrak{A}$ называется гипериммунным, если неверно, что для любого натурального числа $n$ найдется суббинитное множество $\mathfrak{A}_{n} \subseteq \mathfrak{A}$, квазисодержащее не менее $n$ попарно различных элементов.

Таким образом, хорошо известное различие между понятиями иммунного и гипериммунного множества по существу сводится к различию между понятиями финитного и субфинитного множества.

2. Некоторые характерные различия конструктивного и «классического» вариантов математического анализа также легко находят свое объяснение в «классической» установке подразумевать конечные множества всех возможных типов финитными. Здесь может быть указана, в частности, теорема об эквивалентности определений интеграла по Риману (в виде предела интегральных сумм) и по Дарбу (в виде сечения в множестве интегралов ступенчатых оценок рассматриваемой функции), с точки зрения конструктивного направления опровергаемая на примере [3]. Основным моментом «классического» доказательства, нарушающимся при этом с конструктивной точки зрения, является именно финитность (заведомо субфинитной) совокупности отрезков интегрального разбиения, содержащих «большие» колебания интегрируемой функции.

\section{Литература}

[1] Veldman $W$. Some intuitionistic variations on the notion of a finite set of natural numbers// Repsectives on negation. Tilburg Univ. Press, Tilburg, 1995. P. 177-202. 
[2] Владимиров А. А. Об иерархиях конечных множеств// ДАН. 2006. Т. 406, № 3. C. 295-297.

[3] Владимиров А. А. О сравнении интегралов Дарбу и Римана в конструктивном математическом анализе// Препринт доступен на сайте http://arxiv.org/abs/ 0911.2892

[4] Владимиров А.А., Домбровский-Кабанченко М.Н. Ступенчатая семантическая система. М.: Изд-во ВЦ РАН, 2009.

[5] Заславский И.Д. Некоторые свойства конструктивных вещественных чисел и конструктивных функций// Труды матем. ин-та им. В. А. Стеклова. Т. 67. C. 385-457.

[6] Кушнер Б. А. Лекции по конструктивному математическому анализу. М.: Наука, 1973

[7] Марков А. А. Попытка построения логики конструктивной математики// Исследования по теории алгорифмов и математической логике. Т. 2. М.: Изд-во ВЦ АН ССCР, 1976. С. 3-31.

[8] Марков А. А., Нагорнъй Н. М. Теория алгорифмов. М.: ФАЗИС, 1996.

[9] Нагорный Н. М. Монолитно ли понятие конечного множества? (По поводу одной непубликовавшейся работы А. А. Маркова)// Марков А. А. Избр. труды. Т. 2. М.: Изд-во МЦНМО, 2003. С. 527-534.

[10] Нагорный Н. М., Шанин Н. А. Андрей Андреевич Марков (к шестидесятилетию со дня рождения)// Успехи матем. наук. 1964. Т. 19, № 3. С. 207-223.

[11] Роджерс $X$. Теория рекурсивных функций и эффективная вычислимость. М.: Мир, 1972. 